\title{
A concise modeling methodology and its application in thermal system
}

\author{
Y. Lu \\ Laboratory of Marine Simulation and Control Dalian \\ Maritime University
}

\author{
X.K. Zhang* \\ Laboratory of Marine Simulation and Control Dalian \\ Maritime University
}

\author{
X.J. Chen \\ Laboratory of Marine Simulation and Control Dalian \\ Maritime University
}

\begin{abstract}
The paper presents an interesting method for the derivation of transfer function from passive RCLnetworks (Resistance, Capacitance and Inductance) with a ladder structure. In this paper, circuit network modeling methodology is extended into thermal system. In order to apply directly the methodology to thermal system, firstly pseudo bond graph method, as an intermediary, is utilized to depict the model of thermal system, secondly the model is transformed into its equivalent circuit diagram, finally according to the methodology, the mathematical model in format of transfer function of thermal system can be rapidly obtained by observing circuit diagram alone. Through a modeling example of heat transfer in building envelope wall, it has been verified that the mathematical models obtained by circuit network modeling methodology and pseudo bond graph method are identical, but this methodology has the advantages of simplicity, convenience, rapidness and intuitiveness in manual derivation of transfer function of thermal system.
\end{abstract}

Keywords- thermal system; circuit network methodology; modelling; bond graph; heat transfer

\section{INTRODUCTION}

The commonly used circuit network modeling method is direct derivation of the transfer function of circuit system based on Kirchhoff's law or firstly converting the circuit system into a signal flow graph, and secondly applying the Mason Gain Formula to derive the transfer function. However, the derivation process of the transfer function is a little bit complex. Compared with the aforementioned methods, the circuit network modeling methodology has the advantages of simplicity, convenience, rapidness and intuitiveness in some respects. By observing the circuit diagram, the mathematical model in format of the transfer function of the moderate complex circuit system can be established without derivation and within one minute, according to the steps and principles summarized through theoretical deduction and under the premises of correct understanding of and good familiarization with the principles and steps. But it is a pity that such an excellent methodology is only applicable to circuit system [1].

Because of the unified notation for different system elements with the same characteristics, as well as the unified manner for mathematic description of different kinds of engineering systems, the bond graph theory [2] has attracted more and more attention in modeling field. For instance, reference [3] is about modeling of analogue circuits, reference [4] is about modeling of hydraulic system, references [5-6] are about the pseudo bond graph models of thermal system, references [7-8] introduce some bond graph modeling examples of thermal systems and references [9-10] are about the application of bond graph modeling method in multiple energy domains. All the above studies have illustrated that the bond graph modeling method is a very effective modeling tool. However, in some cases, the derivation process of certain kind mathematical model of the system is slightly burdensome. Because of the capability of circuit network modeling methodology in directly obtaining the mathematical model in format of transfer function of the circuit system by observing the circuit diagram, and the simplicity of bond graph method in depicting the bond graph of thermal system, in addition with the achievement of successful conversion of different system elements with the same characteristics as shown in reference [10], it becomes very convenient for us to firstly depict the bond graph of thermal system, then transform it into its equivalent circuit diagram, and ultimately derive the mathematical model of thermal system by utilizing circuit network modeling methodology and vice versa. Furthermore, it has been demonstrated that the aforementioned modeling method can also be applied to the modeling of mechanical translation system [11], mechanical rotation system [12] and hydraulic system [13]. Inspired by the aforementioned three references, hence the paper is 
organized to generalize circuit network modeling methodology to the thermal process. In addition, it may contribute to the system controller design on the basis of some control algorithm [14-15], such as the robust control algorithm, after obtaining the transfer function of system.

\section{PSEUDO BOND GRAPH MODELING METHOD OF THERMAL SYSTEM}

The bond graph modeling method of thermal system is analogous to the bond graph modeling method of electric system, wherein temperature parallels voltage; heat flow parallels current; thermal resistance and thermal capacitance correspond to resistor and capacitor respectively. However, there is no thermal inertial element that resembles inductor. There is also no specific power supply element, but the objet or point with the highest temperature can be taken as an effort source or energy flow source. It should be noted that the product of temperature and heat flow is no longer power. Therefore, Karnopp put forward the concept of pseudo bond graph. In pseudo bond graph, the combinations of temperature $(T)$ and heat flow $(\dot{Q})$, or pressure $(P)$ and mass flow $(\dot{m})$ are commonly utilized as the effort variable and flow variable, whose product is no longer the power in true sense, but the derivation process of state space equations still follows the general rules of bond graph. The reference [6] points that the main approaches to thermal modeling are of two types: energy (true) and pseudo bond graph. Comparing with energy bond graph, pseudo bond graph has several advantages for thermal modeling, such as constitutive relationships of the basic thermal components are linear in nature and basic thermal process of heat flow and heat storage is generally expressed in pseudo-bond graph.

\section{A. Thermal System Modeling Methodology}

The pseudo bond graph modeling method of thermal system is utilized to model the heat transfer through a homogeneous wall which constitutes the envelope of the building. In this kind of cases, the lumped parameter assumption is usually adopted; therefore the wall can be separated into a number of layers as the literature [5] does, which stores and conducts heat simultaneously by conduction. In addition, there is also the convectional phenomenon among the external layers, the inside and outside surroundings. The wall of building envelope is taken as an example throughout this paper for the detailed demonstration of the methodology that we propose.

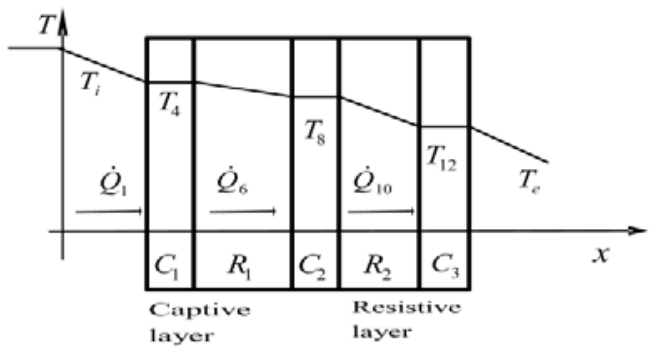

FIGURE I. SKETCH OF THE BUILDING ENVELOPE WALL.

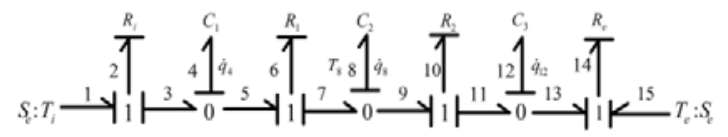

FIGURE II. NUMBERED PSEUDO BOND GRAPH OF THE WALL.

\section{B. Obtain Simplified State Space Equations of Thermal System}

The state space equations can be obtained and simplified only if the certain steps are followed [11]. The simplified state space equations of thermal system are as shown by equation (1).

$$
\begin{gathered}
\left(\begin{array}{c}
\dot{q}_{4} \\
\dot{q}_{8} \\
\dot{q}_{12}
\end{array}\right)=\left(\begin{array}{ccc}
-\left(\frac{1}{C_{1} R_{i}}+\frac{1}{C_{1} R_{1}}\right) & \frac{1}{C_{2} R_{1}} & 0 \\
\frac{1}{C_{1} R_{1}} & -\left(\frac{1}{C_{2} R_{1}}+\frac{1}{C_{2} R_{2}}\right) & \frac{1}{C_{3} R_{2}} \\
0 & \frac{1}{C_{2} R_{2}} & -\left(\frac{1}{C_{3} R_{2}}+\frac{1}{C_{3} R_{e}}\right)
\end{array}\right)\left(\begin{array}{c}
q_{4} \\
q_{8} \\
q_{12}
\end{array}\right)+\left(\begin{array}{cc}
\frac{1}{R_{i}} & 0 \\
0 & 0 \\
0 & -\frac{1}{R_{e}}
\end{array}\right)\left(\begin{array}{l}
T_{i} \\
T_{e}
\end{array}\right) \\
T_{8}=\left(\begin{array}{lll}
0 & \frac{1}{C_{2}} & 0
\end{array}\right)\left(\begin{array}{l}
q_{4} \\
q_{8} \\
q_{12}
\end{array}\right)
\end{gathered}
$$

The derivation of transfer function in symbolic form from state space equations (1) is as shown by equation (2). Therefore, the output of the system $T_{8}$, namely temperature of $C_{2}$, which is randomly selected and difficult to measure in reality, can be obtained as shown by equation (3).

$\left[\begin{array}{ll}G_{i}(s) & G_{e}(s)\end{array}\right]=\left(\begin{array}{lll}0 & \frac{1}{C_{2}} & 0\end{array}\right)\left(s I-\left(\begin{array}{ccc}-\left(\frac{1}{C_{1} R_{i}}+\frac{1}{C_{1} R_{1}}\right) & \frac{1}{C_{2} R_{1}} & 0 \\ \frac{1}{C_{1} R_{1}} & -\left(\frac{1}{C_{2} R_{1}}+\frac{1}{C_{2} R_{2}}\right) & \frac{1}{C_{3} R_{2}} \\ 0 & \frac{1}{C_{2} R_{2}} & -\left(\frac{1}{C_{3} R_{2}}+\frac{1}{C_{3} R_{e}}\right)\end{array}\right)\right)^{-1}\left(\begin{array}{cc}\frac{1}{R_{i}} & 0 \\ 0 & 0 \\ 0 & -\frac{1}{R_{e}}\end{array}\right)$

$$
\left[G_{i}(s) \quad G_{e}(s)\right]=\left(\begin{array}{c}
\frac{R_{1} R_{2}\left(R_{e}+s C_{3} R_{2} R_{e}+R_{2}\right)}{\left(R_{1}+s C_{1} R_{1} R_{i}+R_{i}\right)\left(R_{2}+s C_{2} R_{1} R_{2}+R_{1}\right)\left(R_{e}+s C_{3} R_{2} R_{e}+R_{2}\right)-R_{i} R_{2}\left(R_{e}+s C_{3} R_{2} R_{e}+R_{2}\right)-R_{1} R_{e}\left(R_{1}+s C_{1} R_{1} R_{i}+R_{i}\right)} \\
\frac{-R_{1} R_{2}\left(R_{1}+s C_{1} R_{1} R_{i}+R_{i}\right)}{\left(R_{1}+s C_{1} R_{1} R_{i}+R_{i}\right)\left(R_{2}+s C_{2} R_{1} R_{2}+R_{1}\right)\left(R_{e}+s C_{3} R_{2} R_{e}+R_{2}\right)-R_{i} R_{2}\left(R_{e}+s C_{3} R_{2} R_{e}+R_{2}\right)-R_{1} R_{e}\left(R_{1}+s C_{1} R_{1} R_{i}+R_{i}\right)}
\end{array}\right)^{\mathrm{T}}
$$

$T_{8}=\left[\begin{array}{ll}G_{i}(s) & G_{e}(s)\end{array}\right]\left(\begin{array}{c}T_{i} \\ T_{e}\end{array}\right)$ 
The derivation process of the mathematical model in format of transfer function of thermal system is a little cumbersome by directly using the state space equations obtained by pseudo bond method, in addition the derivation process of the state space equations itself is also a little bit burdensome. However, the mathematical model in format of transfer function can be established directly and easily by observing its equivalent circuit diagram alone on the basis of the circuit network modeling methodology.

\section{Circuit NETWORK MODELING METHODOLOGY}

On the basis of signal flow graph and Mason's gain formula, Wang and Ji [1] summarized and put forward the circuit network modeling methodology, which makes it simpler, more convenient, more rapid and more intuitive in the derivation of mathematical model in format of the transfer function of the circuit network system compared with the Mason's gain formula or the Kirchhoff's law. The methodology is suitable for the analysis of circuit network systems powered by single, multiple or combined voltage source and current source.

The circuit network modeling procedures are composed of the following steps [1]:

1) In the circuit network system, mark grounded node (using 0 for representation), input nodes (using $i(n)$ for representation, wherein $n$ is the number of input nodes), as well as other sequentially numbered active nodes. If there is no ground point, grounded node should be selected subjectively. It also should be noted that only one voltage source and input node can be taken as effective at the same time, that is to say when one voltage source is considered effective; other voltage sources should be treated as short circuit; Then according to the principles of superposition, the output of the circuit system can be obtained, wherein the different directions of current should be taken into account.

2) How many frontal channels could be found out from input node to output node on the circuit network system, how many product terms there are to be added in the numerator. The frontal channel is a channel from input node to output node without encountering grounded node, and any active node cannot be encountered more than once.

3) Each above-mentioned product term is composed of the product of two parts. The first part is the admittance product of all circuit elements that the frontal channel encounters, and the second part is the "multiple expansions" of the self-admittance of those active nodes that the frontal channel does not encounter. If the frontal channel encounters all active nodes, the second part is equal to one. In order to make the writing of "multiple expansions" more convenient and more concise, rules of the abbreviated formula of "multiple expansions" are established. Draw a horizontal line under the common terms when the self-admittance product contains any common terms. For example:

$$
\begin{aligned}
& (a+\underline{b})(b+c)=(a+b)(b+c)-b^{2} \\
& (a+\underline{b)}(b+c)(d+e) \\
& =(a+b)(b+c)(d+e)-b^{2}(d+e)
\end{aligned}
$$

The common term can be obtained by observing the circuit diagram. It is the admittance of the only public element sandwiched between two active nodes. The sign of "multiple expansions" is negative when expanded odd times, positive for even times.

4) As for the denominator, it equals the "multiple expansions" of self-admittance product of all active nodes.

If there are 3 active nodes or more in the circuit diagram, the 5 th rule and 6 th rule may be used, which are omitted in this paper. For detailed information of the two rules and deeper understanding of circuit network modeling methodology, please refer to the reference [1].

\section{TRANSFORM THE PSEUDO BOND GRAPH INTO ITS EQUIVALENT CIRCUIT DIAGRAM}

Transform the pseudo bond graph in Figure 2 into its equivalent circuit diagram, and then apply the circuit network methodology to directly write out its mathematical model, which may take less than one minute and can avoid the trouble of the derivation process. Figure 3 shows the equivalent circuit diagram corresponding to Figure 2. As long as familiar with the physical meaning of each element of pseudo bond graph, it is not difficult to complete the transformation within one minute.

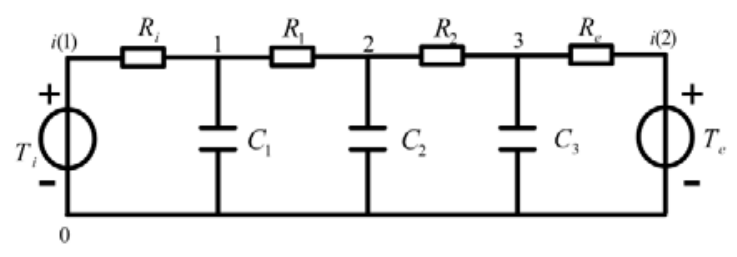

FIGURE III. EQUIVALENT CIRCUIT DIAGRAM OF THERMAL SYSTEM.

For the above circuit network system, grounded node 0 , input nodes $i(1)$ and $i(2)$, active nodes 1,2 and 3 are as shown in Figure 3. In this case the active node 2 is also regarded as the output node. Therefore, there is only one frontal channel for each input node as shown 
in Figure 3, which are $i(1) \rightarrow R_{i} \rightarrow 1 \rightarrow R_{1} \rightarrow 2$ and $i(2) \rightarrow R_{e} \rightarrow 3 \rightarrow R_{2} \rightarrow 2$ respectively. According to the 6 basic rules of the circuit network modeling methodology (in this case only the former 4 rules are

$$
T_{8}=\frac{\frac{1}{R_{i}} \frac{1}{R_{1}}\left(\frac{1}{R_{2}}+s C_{3}+\frac{1}{R_{e}}\right) T_{i}-\frac{1}{R_{e}} \frac{1}{R_{2}}\left(\frac{1}{R_{i}}+s C_{1}+\frac{1}{R_{1}}\right) T_{e}}{\left(\frac{1}{R_{i}}+s C_{1}+\frac{1}{\underline{R_{1}}}\right)\left(\frac{1}{R_{1}}+s C_{2}+\frac{1}{\underline{R_{2}}}\right)\left(\frac{1}{R_{2}}+s C_{3}+\frac{1}{R_{e}}\right)}
$$$$
=\frac{\frac{11}{R_{i} R_{1}}\left(\frac{1}{R_{2}}+s C_{3}+\frac{1}{R_{e}}\right) T_{i}-\frac{1}{R_{e} R_{2}}\left(\frac{1}{R_{i}}+s C_{1}+\frac{1}{R_{1}}\right) T_{e}}{\left(\frac{1}{R_{i}}+s C_{1}+\frac{1}{R_{1}}\right)\left(\frac{1}{R_{1}}+s C_{2}+\frac{1}{R_{2}}\right)\left(\frac{1}{R_{2}}+s C_{3}+\frac{1}{R_{e}}\right)-\frac{1}{R_{1}^{2}}\left(\frac{1}{R_{2}}+s C_{3}+\frac{1}{R_{e}}\right)-\frac{1}{R_{2}^{2}}\left(\frac{1}{R_{i}}+s C_{1}+\frac{1}{R_{1}}\right)}
$$

$R_{1} R_{2}\left(R_{e}+s C_{3} R_{2} R_{e}+R_{2}\right) T_{i}-R_{1} R_{2}\left(R_{1}+s C_{1} R_{1} R_{i}+R_{i}\right) T_{e}$ utilized), it is very convenient to directly write out the system output of its equivalent circuit diagram as shown in Figure 3:
It is extremely obvious that Equation (3) and Equation (4) are identical; therefore the circuit network modeling methodology is absolutely applicable to thermal system modeling. In some extent, the methodology used by Equation (4) is pretty simpler and more rapid. It is apparent that there is no need for this methodology to rely on other software, 20sim for example. In addition, it is very convenient to derive the transfer function of thermal system from the circuit diagram by taking the advantages of the circuit network methodology, which makes it easy to design the temperature controller of building. Conversely, it is also not difficult to depict the circuit diagram of the thermal system based on its transfer function. Thus it may be very convenient for us to manufacture circuit board equivalent to the circuit diagram, which is composed of resistor and capacitor only. Thereafter, it is easy to form a semi-physical simulation system by making use of the computer to control the circuit board just like the way shown in reference [9].

\section{CONCLUSIONS}

In this paper, the network modeling methodology is successfully extended into the thermal system, wherein the pseudo bond graph is treated as an intermediary. The pseudo bond graph of thermal system is firstly depicted, and then converted into its equivalent circuit diagram, and ultimately the mathematical model in format of transfer function of thermal system is directly written out according to the circuit network methodology. Furthermore, it has been validated by the thermal system modeling example that the proposed method is rapider to some extent when it comes to manually derive the transfer function of the thermal system. In addition, it can also deal with the multiple heat source thermal system, and get the temperature characteristics of the part of the system that does not facilitate to measure. The methodology presented in this paper also has wide guide significance on the semi physical simulation and temperature controller design.

\section{ACKNOWLEDGMENT}

This work is partially supported by the National Natural Science Foundation of China (Grant No. 51109020) and the Fundamental Research Funds for the Central Universities (No.3132014302).

\section{REFERENCES}

[1] Huixian Wang, Zhiwen Ji, A new method for linear electrical, electronic and mechanical network calculation, Dalian Maritime University press: Dalian, 1997.

[2] H.M. Paynter, Hydra ulic by analog-an electronic model of a pumping plant, Boston Society of Civil Engineering, Vol.7, pp. 197$219,1959$.

[3] W. Denman, M.H. Zaki, S. Tahar, Formal verification of bond graph modeled analogue circuits, IET Circuits, Devices \& Systems, 5(3), pp. 243-255, 2011.

[4] Lee S J, Chang P H, Modeling of a hydraulic excavator based on bond graph method and its parameter estimation, Journal of mechanical science and technology, 26(1), pp. 195-204, 2012.

[5] Abdelatif MERABTINE, Riad BENELMIR, Mohammed EL GANAOUI, Pseudo-Bond Graph Model for the Analysis of the thermal behavior of building, Thermal Science, 17(3), pp. 723-732, 2013.

[6] Surjya K. Pal, Derek A. Linkens, Temperature distribution in steel during hot rolling: pseudo-bond graph view, Simulation Modelling Practice and Theory, 10(1), pp. 69-85, 2002.

[7] J.L. Baliño, Galerkin finite element method for incompressible thermofluid flows framed within the bond graph theory, Simulation Modelling Practice and Theory, 17(1), pp. 35-49, 2009.

[8] K. Medjaher, A.K. Samantaray, B. Ould Bouamama, Bond graph model of a vertical U-tube steam condenser coupled with a heat exchanger, Simulation Modelling Practice and Theory, 17(1), pp. 228-239, 2009.

[9] Abd Essalam Badoud, Mabrouk Khemliche, Belkacem Ould Bouamama, et al., Bond graph modeling and optimization of photovoltaic pumping system: Simulation and experimental results, Simulation Modelling Practice and Theory, Vol.36, pp. 84-103, 2013.

[10] Alabakhshizadeh, Y. Iskandarani, G. Hovland, et al., Analysis, Modeling and Simulation of Mechatronic Systems using the Bond Graph Method, Modeling identification and control, 32(1), pp. 35-45, 2011

[11] Xianku Zhang, Yicheng Jin, Modeling of Control System and Digital Simulation, second ed., Dalian Maritime University press: Dalian, 2013. 
[12] Xianku Zhang, Ge Guo, Network modeling method of mechanical rotation system, Mechanical science and technology. 26(2), pp. 265$268,2007$.

[13] Xianku Zhang, Network modeling method of hydraulic system, Machine tool and hydraulics, 34(12), pp. 105-107,109, 2006.

[14] Shaikh P H, Nor N B M, Nallagownden P, et al., A review on optimized control systems for building energy and comfort management of smart sustainable buildings, Renewable and Sustainable Energy Reviews, Vol.34, pp. 409-429, 2014.

[15] Nassif N, Modeling and optimization of HVAC systems using artificial neural network and genetic algorithm. Building Simulation, 7(3): pp. 237-245, 2014. 